\title{
Los Acuíferos de nuestro país: un tesoro para las generaciones venideras
}

\author{
Antonela Peralta •; Estela Mónica López Sardi ••
}

\section{Resumen}

Puesto que las aguas de la superficie son tangibles y se han invertido importantes sumas de dinero en construir represas, diques, embalses artificiales, acueductos y canales de riego, todas obras visibles, resulta lo más natural que se piense que esta manifestación del agua constituye la mayor fuente para satisfacer las necesidades del mundo. Sin embargo, los ríos y lagos constituyen menos del $3 \%$ del agua dulce fluida de nuestro planeta. E1 97\% restante (unos $1230 \mathrm{~km}^{3}$ de agua) se encuentran en el subsuelo. En el presente artículo se describe en forma general la estructura de los acuíferos y se analiza la situación de los acuíferos que se encuentran en el territorio de la República Argentina.

Palabras clave: acuíferos, aguce, agua potable.

\begin{abstract}
The surface waters are tangible. There are important dams, dikes, reservoirs, aqueducts and irrigation canals, all of them visible works. But those aren't the biggest water sources to satisfied the world needs. They are only visible. However, the rivers and lakes constitute less than $3 \%$ of fresh water flowing from our planet. The remaining $97 \%$ (about $1230 \mathrm{~km} 3$ of water) is groundwater. The present article describes the general structure of aquifers and analyzed the situation of aquifers that are in the territory of Argentina.
\end{abstract}

Keywords: aquifers, freshwater, potable water.

Fecha de Recepción: diciembre 2011 | Fecha de aceptación: marzo 2012

- Becaria del Proyecto de Investigación "Técnicas de Potabilización de agua en zonas de catástrofe o situaciones de emergencia”. EST / IESE.

-• Directora del Proyecto de Investigación "Técnicas de Potabilización de agua en zonas de catástrofe o situaciones de emergencia”. EST / IESE. 


\section{¿Qué es un acuífero?}

Básicamente, un acuífero es un depósito de agua subterránea. El agua de las precipitaciones, absorbida por el suelo, rellena las cavidades en la arena, arcilla, grava o piedras del subsuelo, donde se almacena. La gravedad provoca el descenso de la masa de agua hasta que se encuentra con una capa impermeable.

Los geólogos denominan litosfera a la corteza terrestre y rocas a todos los materiales que la componen, ya sean éstos no consolidados como las arenas y las arcillas o consolidados como el granito y la caliza.

A mayor o menor profundidad, todos los materiales de la corteza terrestre son normalmente porosos. Esta parte se denomina zona de fracturación. Los poros o aberturas de esta parte de la litosfera, pueden encontrarse parcial o totalmente saturados de agua.

El estrato superior, en donde las aberturas están sólo parcialmente llenas de agua se denomina "zona de aireación o no saturada". Inicialmente por debajo de ésta, y en donde esas aberturas se hallan completamente llenas de agua, está la "zona de saturación".

La franja de agua contenida en el suelo tiene una particular importancia para la agricultura puesto que es la que suministra el agua necesaria para el crecimiento de las plantas. Su espesor varía de acuerdo con los tipos de suelos y de la vegetación y puede extenderse desde unos pocos metros hasta varios de ellos por debajo de la superficie del terreno.

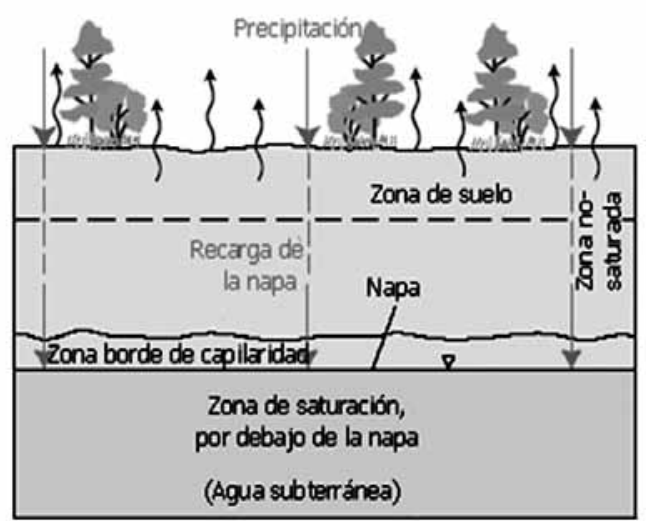

Fig.1: Distribución subsuperficial del agua.

El agua que pasa a través de la capa de humedad del suelo llega a la franja intermedia o de recarga y continúa su descenso por acción gravitacional. El agua 
suspendida en esta franja constituye un almacenamiento fósil, puesto que no se puede recuperar para utilizarla. Su utilidad consiste únicamente en proveer de agua a la zona de saturación.

La franja capilar yace inmediatamente por debajo de la intermedia y encima de la zona de saturación. Su espesor y la cantidad de agua que retiene dependen del tamaño de los granos del material.

La superficie que separa la franja capilar de la zona de saturación recibe el nombre de nivel freático.

El agua contenida en la "zona de saturación" es la única parte de toda el agua del subsuelo de la cual se puede hablar con propiedad como agua subterránea. La zona de saturación, podría asimilarse a un gran embalse natural o sistema de embalses cuya capacidad total es equivalente al volumen conjunto de los poros o aberturas de las rocas que se hallan llenas de agua y su espesor varía desde unos pocos metros hasta varios cientos.

El agua subterránea se puede encontrar en forma de un solo cuerpo continuo o también en estratos separados.

Se denominan acuíferos a aquellas formaciones o estratos comprendidos dentro de la zona de saturación, que son capaces de suministrar agua a pozos y manantiales, los cuales a su vez, sirven como fuentes prácticas de abastecimiento del líquido. Otros términos se usan para denominarlos, son formación almacenadora y depósito de agua subterránea.

Para que una formación geológica pueda ser calificada de acuífero, debe contener poros o intersticios llenos de agua y suficientemente grandes como para que permitan que el agua se desplace hacia pozos y manantiales a caudal apreciable. Esta formación puede estar conformada por materiales muy variados como gravas de río, calizas muy agrietadas, areniscas porosas poco cementadas, arenas de playa, algunas formaciones volcánicas, depósitos de dunas, etc.

Si admitimos que los acuíferos reciben agua de la precipitación (aunque puede recibirla por otras vías), se pueden definir tres zonas: zona de alimentación $o$ recarga, zona de circulación y zona de descarga.

La zona de alimentación es aquella donde el agua de precipitación se infiltra. La zona de descarga es la zona donde el agua sale del acuífero, como puede ser un manantial o la descarga al mar o a un río. La zona de circulación es la parte comprendida entre la zona de alimentación y la zona de descarga.

Dos son las funciones importantes que realiza un acuífero, por un lado almacena agua, sirviendo como depósito y por otro, transmite agua como lo hace un conducto. Esto se debe a que las aberturas o poros de una formación acuífera sirven tanto de espacio para almacenamiento, como de red de conductos. El agua subterránea se mueve constantemente a través de distancias extensas y desde las áreas de recarga hacia las de descarga. El desplazamiento es muy lento, con 
velocidades que se miden en metros por día y a veces en metros por año. Como consecuencia de ello y del gran volumen que su porosidad representa, un acuífero retiene enormes cantidades de agua en almacenamiento inestable.

En los últimos años, la investigación hidrogeológica se ha centrado en los problemas de la calidad de agua subterránea. En la mayoría de los casos, no se trata ya de "encontrar agua", sino de estudiar cómo la calidad de agua subterránea se ha visto afectada por actividades humanas, predecir la evolución del problema, intentar paliarlo, o, en un caso más afortunado, simplemente adoptar las medidas oportunas para que estos problemas no lleguen a producirse.

La mala calidad del agua subterránea puede ser debida a causas naturales o a la actividad humana. En general, al hablar de contaminación nos referimos a esta última, por ejemplo, un vertido industrial, pero en muchas ocasiones, la distinción no es fácil pues una actividad humana no contaminante (en general, los bombeos) también altera un equilibrio previo, provocando el deterioro la calidad del agua subterránea.

Las vías por las que distintas sustancias llegan a los acuíferos contaminando las aguas subterráneas son muy diversas:

- Infiltración de sustancias depositadas en superficie o de la lluvia a través de ellas.

- Filtración de sustancias almacenadas bajo tierra, o disolución de ellas por el agua subterránea.

- Filtración desde un río influente.

- Derrames o rezumes accidentales de depósitos o conducciones, superficiales o enterrados.

- Desde la superficie, a través de captaciones abandonadas o mal construidas.

- Desde otro acuíferos, a través de las captaciones.

- Inyecciones en pozos. En ocasiones ocultamente, otras veces tras un minucioso estudio técnico.

Por la mayoría de estas vías, los contaminantes alcanzan la superficie freática más superficial, y posteriormente re-difunden en el acuífero, siendo transportados por el flujo subterráneo.

Las principales actividades humanas que generan contaminación de las aguas subterráneas son: disposición de residuos sólidos urbanos y de aguas residuales, actividades agrícolas y ganaderas, las actividades industriales y la minería. 


\section{Los acuíferos de la Argentina}

En un mundo en el que agua se está transformando en un recurso estratégico, Sudamérica y en particular la Argentina, están en condiciones de abastecer de agua potable al mundo por varios siglos.

El Acuífero Guaraní (AG) constituye uno de los reservorios subterráneos de agua dulce más importantes del mundo, con una reserva estimada entre 40.000 y $50.000 \mathrm{~km} 3$, volumen suficiente para abastecer a la población mundial actual (6.000 millones) durante unos 200 años, a una tasa de 100 litros/día por habitante. Se desarrolla en el ámbito de la cuenca del Río Paraná en alrededor de 1.190.000 km2 y es compartido en orden de extensión territorial por Brasil, Argentina, Paraguay y Uruguay.

Brasil abarca una superficie de aproximadamente $850.000 \mathrm{~km} 2(9,9 \%$ del $225.000 \mathrm{~km} 2(7,8 \%)$, en Paraguay $70.000 \mathrm{~km} 2(17,2 \%)$ y en Uruguay $45.000 \mathrm{~km} 2$ $(25,5 \%)$. Las profundidades son muy variables, entre los 50 y 1.500 metros.

Con respecto a su explotación, en Brasil se emplean unos 500 pozos para el abastecimiento total o parcial de 300 ciudades de entre 5.000 y 500.000 habitantes; también se lo utiliza para la industria, el riego y la bañoterapia.

En Uruguay están operando unos 135 pozos, con caudales en surgencia de hasta $400 \mathrm{~m} 3 / \mathrm{h}$ y con bombeo hasta $1.000 \mathrm{~m} 3 / \mathrm{h}$. El agua se emplea para abastecimiento humano, riego, industria, secado de grano, bañoterapia y control de heladas.

En Argentina, el uso está restringido a 7 perforaciones de 1.000 a $1.300 \mathrm{~m}$, situadas en Entre Ríos, que se emplean para bañoterapia y otras más someras, de menos de $200 \mathrm{~m}$, en áreas donde el acuífero aflora o se ubica a poca profundidad (Misiones y Corrientes), que se utilizan para el abastecimiento humano y el riego.

En Paraguay se lo emplea para el abastecimiento de pequeñas comunidades (menos de 4.000 habitantes) con caudales escasos, dado que se capta sólo la sección superior del mismo.

El Sistema Acuífero Toba (SAT) abarca en la República Argentina una superficie aproximada de $210.000 \mathrm{~km} 2$, correspondiendo a un 7,7\% de la superficie continental del territorio nacional. Incluye a las provincias de Salta, Jujuy, Tucumán, Formosa, Chaco y Santiago del Estero.

No se conoce el número total de pozos perforados en el Sistema Acuífero Toba, sin embargo, a continuación se expresan algunas cifras orientativas. En el Chaco Boreal Salteño se han censado 447 pozos, pero en la región el número se estima que debe superar los 500. En el Chaco Austral Salteño se estima que existen otras 750 perforaciones y en la región de Termas de Río Hondo se han perforado aproximadamente 4.000 pozos. 
El Sistema Acuífero Yrenda (SAY) es un sistema hidrogeológico regional que abarca cerca de dos tercios de la Región Occidental del Paraguay y parte del chaco Argentino y Boliviano. Este acuífero incluye al "Acuífero Toba" mencionado más arriba. Es un acuífero formado por sedimentos cuaternarios y terciarios no consolidados de la Formación Chaco. La salinidad de su agua subterránea aumenta a lo largo de la dirección de flujo que es de oeste a este y su velocidad se estima en el rango de 20 a $46 \mathrm{~m} /$ año. La recarga de este sistema acuífero tiene lugar en la región boliviana a través de la infiltración directa de precipitación y agua del río, en las colinas sub-andinas (serranía Aguaragüe).

\section{Proyectos de protección de acuíferos en América y Argentina}

Importantes organismos nacionales e internacionales han estado trabajando en los últimos años en proyectos dedicados a la protección y preservación de los acuíferos. A continuación se mencionan los de mayor relevancia:

- El proyecto Marco para la Gestión Sostenible de los Recursos Hídricos de la Cuenca del Plata: está siendo desarrollado por los gobiernos de la Argentina, Bolivia, Brasil, Paraguay y Uruguay, busca identificar prioridades de acción coordinada y establecer un marco de adaptación a los riesgos crecientes que ocasionan las grandes inundaciones y las sequías (eventos originados en los efectos de El Niño), así como prevenir la contaminación originada en las cargas sedimentarias excesivas en el estuario de La Plata.

- El Proyecto para la Protección Ambiental y Desarrollo Sostenible del Sistema Acuífero Guaraní (SAG): se está llevando a cabo entre los gobiernos de Argentina, Brasil, Paraguay y Uruguay y es cofinanciado por el Fondo Mundial para el Medio Ambiente (GEF). El Banco Mundial actúa como operador del fondo y la Organización de Estados Americanos (OEA) es la Agencia Ejecutora. Las contrapartes nacionales son atendidas con aportes en servicios, infraestructura y personal de las reparticiones involucradas. El SAG es el primer proyecto de las Américas dedicado a los acuíferos transfronterizos y una de las primeras iniciativas del mundo en la que varios países están involucrados.

- La OEA/UDSMA, además, conjuntamente con el Programa Hidrológico Internacional (PHI) de la UNESCO, está ayudando a coordinar el desarrollo y ejecución en las Américas del Programa UNESCO/OEA ISARM2 Américas (Acuíferos Transfronterizos de las Américas) que apunta a mejorar la comprensión y el intercambio y comunicación en temas científicos, 
socioeconómicos, legales, institucionales y ambientales relacionados con el manejo de los acuíferos transfronterizos.

\section{Normativa Internacional aplicable a los acuíferos}

Durante las últimas décadas los organismos nacionales e internacionales especializados en protección ambiental y desarrollo sustentable han elaborado diversas normativas, que se han aplicado al cuidado y manejo de los acuíferos. De esta forma, se ha comenzado a generar un marco normativo, que debe ser respetado tanto por los Estados que poseen estos recursos hídricos en su territorio, como así también por el resto de la comunidad internacional.

A continuación, se presenta una síntesis de dichas normativas y sus puntos de mayor relevancia:

- Resolución 1803 (XVII) de la Asamblea General de las Naciones Unidas, relativa a la soberanía permanente sobre los recursos naturales aprobada el 14 de diciembre de 1962.

- Resolución 63/124 aprobada por la Asamblea General sobre el derecho de los acuíferos transfronterizos. La misma fue sancionada en el 11 de diciembre de 2008 y menciona los factores de mayor importancia para la protección de los acuíferos transfronterizos por parte de los Estados que los poseen.

- Declaración de la Conferencia de las Naciones Unidas sobre el Medio Ambiente Humano, Estocolmo. Fue elaborada entre del 5 al 16 de junio de 1972 con el objetivo de ofrecerle a los pueblos del mundo inspiración y guía para preservar y mejorar el medio ambiente.

- Declaración de Río de Janeiro sobre el Medio Ambiente y Desarrollo. Fue elaborada del 3 al 14 de junio 1992, con el objetivo de establecer una alianza mundial nueva y equitativa mediante la creación de nuevos niveles de cooperación entre los Estados, los sectores claves de las sociedades y las personas y procurando alcanzar acuerdos internacionales en los que se respeten los intereses de todos y se proteja la integridad del sistema ambiental y de desarrollo mundial.

- Cumbre sobre Desarrollo Sostenible en las Américas, de Santa Cruz de la Sierra. Se llevó a cabo entre del 7 al 8 de diciembre de 1996. En la misma, se asumió el compromiso de poner en ejecución el primer Plan de Acción para el Desarrollo Sostenible de las Américas con base en los principios de la Declaración de Santa Cruz de la Sierra, con el propósito de superar los más apremiantes problemas que afrontan nuestros pueblos y asegurar un nivel de vida adecuado y digno para las generaciones presentes y futuras. 
- Cumbre Mundial sobre Desarrollo Sostenible de Johannesburgo. Se llevó a cabo del 2 al 4 e septiembre de 2002 con el objetivo de tratar las problemáticas ambientales y sociales existentes a nivel mundial, de forma de poder hallar un desarrollo sostenible a las actividades que realiza el hombre.

- Tratado de la Cuenca del Plata. fue realizado entre los Gobiernos de las Repúblicas de Argentina, Bolivia, Brasil, Paraguay y Uruguay, en Brasilia, el 22 y 23 de abril de 1960 para mancomunar esfuerzos con el objeto de promover el desarrollo armónico y la integración física de la Cuenca del Plata y de sus áreas de influencia directa y ponderable.

- Acuerdo Marco sobre Medio Ambiente del MERCOSUR. Fue realizado en Asunción el 21 de junio de 2001 entre la República Argentina, la República del Brasil, la república del Paraguay y la República Oriental del Uruguay con objetivo de cooperar con la protección del medio ambiente y la utilización sustentable de los recursos naturales, con vistas a alcanzar una mejor calidad de vida y un desarrollo económico, social y ambiental sustentable.

- Acuerdo sobre el Acuífero Guaraní. El 2 de agosto de 2010, en la provincia de San Juan, República Argentina, se firmo un acuerdo entre la República Federativa del Brasil, la República del Paraguay, la República Argentina y la República Oriental del Uruguay, con el propósito de ampliar el alcance de sus acciones concertadas para la conservación y aprovechamiento sustentable de los recursos hídricos transfronterizos del Sistema Acuífero Guaraní, que se encuentra localizado en sus territorios.

\section{Conclusiones}

El agua es de vital importancia para el desarrollo de todos los procesos fisiológicos del hombre, el crecimiento del las plantas, el desarrollo nacional y aumento del nivel de vida de sus habitantes, entre otras necesidades.

El agua subterránea está en una situación de riesgo creciente, debido a factores tales como el crecimiento constante de la demanda de agua en mega conglomerados urbanos, la explotación descontrolada y la contaminación de los manantiales.

A pesar de este riesgo, hay fundamentos para cierto optimismo. La base tecnológica y científica de la que se dispone para el manejo de los sistemas de aguas subterráneas es cada vez mejor y adicionalmente, la toma de conciencia entre los ciudadanos y las partes interesadas sigue aumentando gracias a la cobertura mediática, los programas de políticas públicas y los numerosos esfuerzos educativos y de difusión de las instituciones nacionales.

Además de los factores anteriormente mencionados, se está trabajando a nivel nacional, regional e internacional en diversos proyectos dedicados a la protección 
y preservación de los acuíferos y se está profundizando cada vez más el marco normativo referente a los mismos.

\section{Fuentes}

Acuerdo Marco sobre el Medio Ambiente del MERCOSUR. Secretaría de Ambiente y Desarrollo Sustentable de la Nación. http://www.medioambiente.gov.ar/archivos/ web/MERCOSUR/File/Acuerdo\%20marco\%20sobre\%20medio\%20ambiente(1). pdf

Acuerdo sobre el Acuífero Guaraní. International Water Law Protect. http://www. internationalwaterlaw.org/documents/regionaldocs/Guarani_Aquifer_AgreementSpanish.pdf

Alfredo fuertes. Proyecto: "Programa marco para la gestión sostenible de los recursos hídricos de la cuenca del plata en relación con los efectos hidrogeológicos de la variabilidad y el cambio climático".Componente: "Aguas subterráneas". Caso de estudio UNESCO/OEA/ISARM/AMERICAS. Sistema acuífero Yrenda-TobaTarijeño Paraguay-Argentina Bolivia. Área: Argentina. INASLA. Diciembre 2004. Arq. Miguel Moreno. Acuífero Guaraní y su explotación como recurso termal. Jornadas sobre Realidades y Potencial del Termalismo en Argentina. Noviembre 2005.

Cumbres de las Américas sobre el Desarrollo Sostenible. Summits of the Americans. http://www.summit-americas.org/summit_sd/summit_sd_poa_sp.pdf

Declaración de Johannesburgo sobre el Desarrollo Sostenible. United Nations. Departament of Economic and Social Affairs. Division of Sustainable Development. http://www.un.org/esa/sustdev/documents/WSSD_POI_PD/ Spanish/WSSDsp_PD.htm

Declaración de Río sobre el Medio Ambiente y el Desarrollo. Naciones Unidas. http://www.un.org/documents/ga/conf151/spanish/aconf15126-1annex1s.htm

El Acuífero Guaraní. www.prodiversitas.bioetica.org/des47.htm

El agua subterránea y los pozos. Libro de referencia dedicado a la industria de los pozos de agua. Primera edición. 1975. Johnson División, UOP Inc. Saint Paul, Minnesota 55165. 
Ongley, E.D. (1997) - Lucha contra la contaminación agrícola de los recursos hídricos. Estudio FAO Riego y Drenaje - 55. www.fao.org.

Organización de las Naciones Unidas para la Educación - $\underline{\text { http://ga.water.usgs.gov/ }}$ edu/watercyclespanish.html

Organización de los Estados Americanos. Unidad de desarrollo sostenible y medio ambiente. El recurso invisible Acuíferos transfronterizos: una oportunidad de cooperación internacional. Series sobre elementos de políticas, número 3. Agosto 2004.

Proyecto Acuífero Guaraní - Proyecto para la Protección Ambiental y Desarrollo Sostenible del Sistema Acuífero Guaraní. Secretaría de Ambiente y Desarrollo sustentable. www.ambiente.gov.ar

Resolución 1803 (XVII) de la Asamblea General sobre la "Soberanía permanente sobre los recursos naturales". Oficina de alto Comisionado de las Naciones Unidas para los Derechos Humanos. http://www2.ohchr.org/spanish/law/recursos.htm

Resolución 63/124 de la Asamblea General de las Naciones Unidas. El derecho de los acuíferos transfronterizos. Documentación de las Naciones Unidas. Guía de Investigación. http://www.un.org/Depts/dhl/resguide/r63sp.shtml

Tratado de la cuenca del Plata. Fondo Financiero para el desarrollo de la Cuenca del Plata(FONPLATA). http://www.fonplata.org/institucional/Documentos\%20 compartidos/tratadocp.pdf 